\title{
THE JACOBSON RADICAL OF THE GROUP ALGEBRA OF A FINITE GROUP
}

\author{
SURINDER SINGH BEDI ${ }^{1}$
}

\begin{abstract}
Let $K$ be a field of characteristic $p \neq 0$ and $G$ a finite group such that $p \mid o(G)$. Suppose $G$ is a Frobenius group with a Sylow $p$-subgroup $P$ as a complement. Then we have proved that
\end{abstract}

$$
J K(G)=\bigcap_{x \in G} J K\left(P^{x}\right) K(G) .
$$

We have given an example to show that equality does not hold in general.

0. Introduction. Let $G$ be a finite group and $K$ a field of characteristic $p \neq 0$. If $p \nmid o(G)$ then $J K(G)=0$ and if $p \mid o(G)$ then $J K(G) \neq 0[2$, Theorem 1.4.1]. If $G$ is a $p$-group then [2, Theorem 2.3.2] $J K(G)=w(K(G))$, the augmentation ideal of $K(G)$. More generally [5, Theorem 16.6], if $G$ is a group having unique Sylow $p$-subgroup $P$, then

$$
J K(G)=w(K(P)) K(G) .
$$

So one asks the following question:

Is $J K(G)=\cap J K(P) K(G)$ where $P$ ranges over Sylow $p$-subgroups of $G$ ? We prove (Theorem 1 and Corollary 1 ) this to be the case for a group $G$ having a normal subgroup $G_{0}$ such that $p \nmid\left(G: G_{0}\right)$ and that $G_{0}$ is a Frobenius group having a Sylow $p$-subgroup $P$ as its complement subgroup. An example due to Passman shows that this equality is false in general.

1. Definitions and preliminaries. A finite group $G$ is said to be a Frobenius group with complement $H$ if $H$ is a subgroup of $G$ such that (1) $\{e\} \subsetneq H \subsetneq G$ and (2) $x H x^{-1} \cap H=(e)$ for every $x \in G-H$. A finite group $G$ is a Frobenius group iff $G$ is isomorphic to a transitive permutation group such that subgroup fixing any of the letters is nontrivial and each permutation $\neq e$ fixes at most one letter [1, Chapter 2, p. 37], [4, p. 57]. The most important fact regarding these groups is the following:

$$
\begin{aligned}
N & =\{x \in G \mid C(x) \cap H=\varnothing\} \cup\{e\} \\
& =\left(G-\bigcap_{x \in G} x H x^{-1}\right) \cup\{e\}
\end{aligned}
$$

Received by the editors April 28, 1978.

AMS (MOS) subject classifications (1970). Primary 16A26, 16A46.

Key words and phrases. Group algebra, Jacobson radical, transitive permutation group, Frobenius group with $p$-Sylow subgroup as complement, Frobenius kernel, central ideal, semidirect product.

${ }^{1}$ The author thanks U.G.C. (India) for financial support. 
is a normal subgroup of order $(G: H)$ and $G=N H$ [1, Theorem 7.5]. The subgroup $N$ is called the Frobenius kernel of $G$. If $H=P$, Sylow $p$-subgroup of $G$ then $p \nmid o(N)$ because $o(N)=(G: P)$. The number of distinct Sylow $p$-subgroups of $G$ equals $o(N)$.

We say $x \in G$ is a $p$-element provided $o(x)=p^{i}$ for some $i \geqslant 0$. The $p$-trace of $\sum a_{x} x \in K(G)$ is defined to be

$$
\operatorname{tr}_{p}\left(\sum a_{x} x\right)=\sum_{x \text { is a } p \text {-element }} a_{x}
$$

LEMMA 1. If $\alpha \in K(G)$ is nilpotent then $\operatorname{tr}_{p}(\alpha)=0$.

For proof see [6, Lemma 2.3.3].

Lemma 2. Suppose $N$ and $H$ are subgroups of the finite group $G$ with $N \triangleleft G$ and $G=N H$. If $\hat{N}=\Sigma_{n \in N} n$, then

$$
J K(H) \hat{N} \subset\left(\bigcap_{x \in G} J K\left(H^{x}\right) K(G)\right) \cap J K(G) .
$$

Proof. Observe that $\hat{N}$ is central in $K(G)$ and $n \hat{N}=\hat{N}$ for all $n \in N$. Let $x \in G=N H$ so $x=n h$, where $n \in N$ and $h \in H$. Then

$$
\begin{aligned}
J K\left(H^{x}\right) \hat{N} & =h^{-1} n^{-1} J K(H) n h \hat{N} \\
& =h^{-1} J K(H) h \hat{N}=J K(H) \hat{N} .
\end{aligned}
$$

Hence $J K(H) \hat{N} \subset \bigcap_{x \in G} J K\left(H^{x}\right) K(G)$. Since $(J K(H) \hat{N}) x=x(J K(H) \hat{N})$ for all $x \in G, J K(H) \hat{N}$ generates a nilpotent two sided ideal of $K(G)$. Thus $J K(H) \hat{N} \subset J K(G)$.

2. Main result. From now on, we assume that $K$ is an arbitrary field of characteristic $p \neq 0, G$ a finite group such that $p \mid o(G)$, and $P$ a fixed Sylow $p$-subgroup of $G$.

TheOREM 1. Suppose $G$ is a Frobenius group with Sylow p-subgroup $P$ as a complement. Then $J K(G)=\bigcap_{x \in G} J K\left(P^{x}\right) K(G)$.

Proof. We shall prove this in two parts by showing that each side equals $J K(P) \hat{N}$ where $N$ is the Frobenius kernel and $\hat{N}$ denotes the element $\Sigma_{x \in N} x$.

First we shall show that $J K(G)=J K(P) \hat{N}$. (Wallace [7] proved, using modular representation theory, that if $G$ is a group such that $G^{\prime} P$ is a Frobenius group with $G^{\prime}$ as the Frobenius kernel and $P$ as a complement then $J K\left(G^{\prime} P\right)=J K(P) \hat{G}^{\prime}$. Our result is stronger and its proof uses simpler

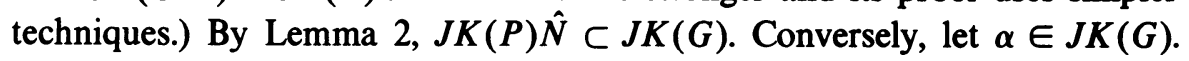
Let

$$
\alpha=\sum_{x \in N} a_{x} x+\sum_{y \in G-N} a_{y} y .
$$

Since $J K(G)$ is nilpotent $\alpha$ is nilpotent. Thus by Lemma 1 , we have $\operatorname{tr}_{p}(\alpha)=$ 0 . Since $p \nmid o(N)$ no element $x \neq 1$ in $N$ is a $p$-element. If $y \in G-N$ then $y$ 
belongs to some Sylow $p$-subgroup of $G$. Thus $y$ is a $p$-element. Therefore $\operatorname{tr}_{p}(\alpha)=0$ gives

$$
a_{1}+\sum_{y \in G-N} a_{y}=0
$$

Since $J K(G) \subset w(K(G))$ we have

$$
\sum_{x \in N} a_{x}+\sum_{y \in G-N} a_{y}=0
$$

and hence $a_{1}=\Sigma_{x \in N} a_{x}$. Now let $n \in N$. Then $\alpha n^{-1} \in J K(G)$ and

$$
\alpha n^{-1}=\sum_{x \in N} a_{x} x n^{-1}+\sum_{y \in G-N} a_{y} y n^{-1} .
$$

Therefore, by the same arguments, $a_{n}=\sum_{x \in N} a_{x}$ and hence $a_{1}=a_{n}$ for all $n \in N$. Let $h \in P$. Then $\alpha h^{-1} \in J K(G)$ and $\alpha h^{-1}=\left(\sum_{x \in N} a_{x h} x h\right) h^{-1}+\alpha^{\prime}$ where support $\left(\alpha^{\prime}\right) \subset G-N$. Then by the above argument, the coefficients of the elements of $N$ in $\alpha h^{-1}$ are all equal. Therefore $a_{h}=a_{n h}$ for all $n \in N$. Hence $\alpha=\sum_{h \in P} a_{h} \hat{N h}$. Since $\alpha \in J K(G) \subset w(K(G))$ we have $\left(\Sigma_{h \in P} a_{h}\right) o(N)$ $=0$ and so $\Sigma_{h \in P} a_{h}=0$ because $o(N) \neq 0$ in $K$. Thus $\Sigma_{h \in P} a_{h} h \in w(K(P))$ $=J K(P)$. Hence $\alpha \in J K(P) \hat{N}$.

Now we shall prove that $\sum_{x \in G} J K\left(P^{x}\right) K(G)=J K(P) \hat{N}$. By Lemma 2, we have

$$
J K(P) \hat{N} \subset \bigcap_{x \in G} J K\left(P^{x}\right) K(G) .
$$

For the reverse inclusion, let $I=\cap_{x \in G} J K\left(P^{x}\right) K(G)$. Let $P_{1}=P$, $P_{2}, \ldots, P_{t}$ be all the distinct Sylow $p$-subgroups of $G$. Then $t \neq 0$ in $K$ because $t=o(N)$. Therefore $I=\bigcap_{i=1}^{t} J K\left(P_{i}\right) K(G)$. Now $\hat{P}_{i}$ annihilates $J K\left(P_{i}\right) K(G)$ on the left so $\hat{P}_{i} I=0 \forall i$. Since $\hat{G}=\hat{N} \hat{P}$ we have $\hat{G} I=0$. Now $G=N \cup P_{1} \cup P_{2} \cup \cdots \cup P_{t}$ where unions are disjoint on the nonidentity elements. Therefore $\hat{G}+t \cdot 1=\hat{N}+\sum_{i=1}^{t} \hat{P}_{i}$ in $K(G)$. But $\hat{P}_{i} I=0 \forall i$ and $\hat{G} I=0$. Therefore $(t \cdot 1-\hat{N}) I=0$. Let $\alpha \in I$. Then $(t \cdot 1-\hat{N}) \alpha=0$ and so $\alpha=\hat{N} \alpha / t$ (because $t \neq 0$ in $K$ ). Therefore $\alpha \in \hat{N} K(G)$. But $G=N P$ and $\hat{N} n=\hat{N} \forall n \in N$. Therefore $\alpha \in \hat{N} K(P)$. Let $\alpha=\hat{N} \beta$ where $\beta \in K(P)$ and $\beta=\Sigma_{y \in P} a_{y} y$. Since $\alpha \in I \subset w(K(G))$ we have $t \cdot\left(\Sigma_{y \in P} a_{y}\right)=0$ and so $\Sigma_{y \in P} a_{y}=0$ (because $t \neq 0$ in $K$ ). Thus $\beta \in w(K(P))=J K(P)$. Hence $\alpha \in$ $\hat{N} J K(P)$. This proves Theorem 1 .

Corollary 1. Let $G$ be a finite group and $p \mid o(G)$. Suppose there exists a normal subgroup $G_{0}$ of $G$ such that $p \nmid\left(G: G_{0}\right)$ and $G_{0}$ is a Frobenius group with $P$ as a complement subgroup. Then $J K(G)=\bigcap_{x \in G} J K\left(P^{x}\right) K(G)$.

Proof. $J K(G)=J K\left(G_{0}\right) K(G)$ [5, Theorem 16.6] and by Theorem 1, $J K\left(G_{0}\right)=\cap_{x \in G_{0}} J K\left(P^{x}\right) K\left(G_{0}\right)$. Hence 


$$
\begin{aligned}
J K(G) & =\left(\bigcap_{x \in G_{0}} J K\left(P^{x}\right) K\left(G_{0}\right)\right) K(G) \\
& =\bigcap_{x \in G_{0}} J K\left(P^{x}\right) K(G)=\bigcap_{x \in G} J K\left(P^{x}\right) K(G) .
\end{aligned}
$$

(The last equality follows from the fact that the Sylow $p$-subgroups of $G$ are precisely those of $G_{0}$.)

Professor Passman in a letter asked if the converse of Corollary 1 holds. He thus asked: If $G$ is a finite group such that $p \mid o(G)$ and $J K(G)=$ $\cap J K(P) K(G)$ as $P$ ranges over Sylow $p$-subgroups of $G$ for some field $K$ of characteristic $p \neq 0$, does there exist in $G$ a normal subgroup $G_{0}$ such that $p \nmid\left(G: G_{0}\right)$ and that $G_{0}$ is a Frobenius group with a Sylow $p$-subgroup as a complement? We give below a generalization (Corollary 2) of Corollary 1. This generalization yields a negative answer to the above question.

Corollary 2. Suppose there exist subgroups $G_{0}, P_{0}$ of the finite group $G$ such that $G \supset G_{0} \supsetneq P \supset P_{0}, G_{0} \triangleleft G, P_{0} \triangleleft G_{0}$ and $G_{0} / P_{0}$ is a Frobenius group with $P / P_{0}$ as $a$ complement. Then $J K(G)=\cap J K(P) K(G), P$ ranging over Sylow p-subgroups of $G$.

Proof. Since $G_{0} / P_{0}$ is a Frobenius group with $P / P_{0}$ as a complement. By Theorem 1 we have

$$
J K\left(G_{0} / P_{0}\right)=\bigcap_{x \in G_{0}} w\left(K\left(P^{x} / P_{0}\right)\right) K\left(G_{0} / P_{0}\right)
$$

Taking the complete preimage of both sides under the canonical map from $K\left(G_{0}\right)$ to $K\left(G_{0} / P_{0}\right)$ we get

$$
J K\left(G_{0}\right)=\bigcap_{x \in G_{0}} J K\left(P^{x}\right) K\left(G_{0}\right)
$$

$$
\begin{aligned}
& J K(G)=J K\left(G_{0}\right) K(G)[5, \text { Theorem 16.6]. Hence } \\
& \qquad \begin{aligned}
J K(G) & =\left(\bigcap_{x \in G_{0}} J K\left(P^{x}\right) K\left(G_{0}\right)\right) K(G) \\
& =\bigcap_{x \in G_{0}} J K\left(P^{x}\right) K(G)=\bigcap_{x \in G} J K\left(P^{x}\right) K(G) .
\end{aligned}
\end{aligned}
$$

ExAMPLE. For $p=2, S_{4}$ satisfies the conditions of Corollary 2, by taking $G_{0}=S_{4}$ and $P_{0}=V_{4}$, but $S_{4}$ does not satisfy the conditions of Corollary 1 . This answers the question of Passman. Of course, now one can ask: Is the converse of Corollary 2 true?

3. Counterexample. The equality $J K(G)=\cap J K(P) K(G)$ where $P$ ranges over $p$-Sylow subgroups of $G$ is false in general, as the following example shows. 
Example (Passman). Let

$$
N=\left\{\left(\begin{array}{ccc}
1 & \alpha & \beta \\
0 & 1 & \gamma \\
0 & 0 & 1
\end{array}\right) \mid \alpha, \beta, \gamma \in Z / 3 Z\right\} .
$$

$N$ is a group of order 27 and $\forall x \in N, x^{3}=1$. It is easy to see that $N$ is generated by $a, b, c$ where

$$
a=\left(\begin{array}{lll}
1 & 1 & 0 \\
0 & 1 & 0 \\
0 & 0 & 1
\end{array}\right), \quad b=\left(\begin{array}{lll}
1 & 0 & 0 \\
0 & 1 & 1 \\
0 & 0 & 1
\end{array}\right) \quad \text { and } \quad c=\left(\begin{array}{lll}
1 & 0 & 1 \\
0 & 1 & 0 \\
0 & 0 & 1
\end{array}\right) ;
$$

$(a, b)=c$ and $c$ is central. Define $\sigma: N \rightarrow N$ by

$$
\sigma\left(\begin{array}{lll}
1 & \alpha & \beta \\
0 & 1 & \gamma \\
0 & 0 & 1
\end{array}\right)=\left(\begin{array}{rrr}
1 & -\alpha & \beta \\
0 & 1 & -\gamma \\
0 & 0 & 1
\end{array}\right)
$$

One checks that $\sigma$ is an automorphism of $N$ of order 2 and $\sigma(a)=a^{2}$; $\sigma(b)=b^{2} ; \sigma(c)=c$. Let $H=\langle x\rangle$ the cyclic group of order 2 .

Let $G=N X_{\sigma} H$ the semidirect product of $N$ by $H$ i.e. $x n=\sigma(n) x \forall n \in$ $N$. $G$ is generated by the elements $x, a^{-1} x a$ and $b^{-1} x b$. So $G$ is generated by the elements of order 2. $o(G)=54$ and $H$ is a Sylow 2-subgroup of $G$. Let $K$ be an algebraically closed field of characteristic 2 . We shall show that $J K(G) \neq \bigcap_{x \in G} J K\left(H^{x}\right) K(G)$. Since $G / N \approx H$ we have $G^{\prime} \subset N$. Also $c=$ $(a, b) \in G^{\prime}, x a=a^{2} x=a(a x)$ so $a \in G^{\prime}$, and $x b=b^{2} x=b(b x)$ so $b \in G^{\prime}$. Thus $G^{\prime}=N$. Hence $G=G^{\prime} H$. Since $c \in G-H$ and $c$ is central we have $N_{G}(H) \neq H$. So $G^{\prime} H$ is not a Frobenius group with $H$ as a complement. Hence by [7, Theorem, p. 103] $J K(G)$ is not central. But we shall show that $\cap_{x \in G} J K\left(H^{x}\right) K(G)$ is central. Let $I=\bigcap_{x \in G} J K\left(H^{x}\right) K(G)$. Now let $x$ be an element of order 2 so that $P=\langle x\rangle$ is a Sylow 2-subgroup of $G$. Then

$$
I \subset J K(P) K(G)=(1+x) K(G)
$$

so $(1-x) I=0$. Since $G$ is generated by elements of order 2 therefore $w(K(G))$ is generated as left ideal by the set $\{(1-x) \mid o(x)=2\}[3$, Lemma 1, p. 153]. Thus $w K(G) I=0$ so $I \subset \hat{G} K(G)[3$, Lemma 2, p. 154]. Hence $I$ is central.

ACKNOWLEDGEMENT. Theorem 1 was proved by me for the case $G=G^{\prime} P$ and $N=G^{\prime}$ and communicated to Professor Passman. He realized the theorem in its present form and also suggested simplification of arguments. We thank him for his contribution and interest in the work. The author is extremely grateful to Dr. R. N. Gupta for his able guidance and constant encouragement during the preparation of this paper. We also thank the referee for his suggestions. 


\section{REFERENCES}

1. D. Gorenstein, Finite groups, Harper and Row, New York, 1968.

2. I. N. Herstein, Noncommutative rings, Carus Math. Mono., No. 15, Math. Assoc. Amer., Wiley, New York, 1968.

3. J. Lambek, Lectures on rings and modules, Blaisdell, Waltham, Mass., 1966.

4. D. S. Passman, Permutation groups, Benjamin, New York, 1968.

5. __ Infinite group rings, Dekker, New York, 1971.

6. __ The algebraic structure of group rings, Wiley, New York, 1977.

7. D. A. R. Wallace, Group-algebras with central radicals, Proc. Glasgow Math. Assoc. 5 (1962), 103-108.

Centre for Advanced Study in Mathematics, Panjab University, Chandigarh-160014, INDIA 